\title{
User Requirement Analysis of Kitchen Storage Products Under the Fusion of Large and Small Data
}

\author{
Kexin $\mathrm{Li}^{\mathrm{a}} \mathrm{b}$, Qun $\mathrm{Wu}^{\mathrm{c}, \mathrm{d}, 1}$ and Zhengning $\mathrm{Nie}^{\mathrm{a}}$ \\ ${ }^{a}$ Art and Design School, Zhejiang Sci-Tech University, Hangzhou (PR China) \\ ${ }^{b}$ Ouhai Research Institute of Zhejiang Sci-Tech University, Wenzhou (PR China) \\ ${ }^{c}$ Collaborative Innovation Center of Culture, Creative Design and Manufacturing \\ Industry of China Academy of Art, Hangzhou (PR China) \\ ${ }^{d}$ Zhejiang Provincial Key Laboratory of Integration of Healthy Smart Kitchen \\ System, Hangzhou (PR China)
}

\begin{abstract}
With the development of online shopping platforms, users are able to express their needs and feelings through online reviews. The analysis of comments from users can help to understand the real needs of users, the iteration of facilitate product and the service transformation of companies. The big data methods can be used to analyse user reviews, which can help designers gain the user needs on a macro perspective, while the traditional small data methods can help designers obtain the capture users' implicit preferences and differential needs. Therefore, the fusion of large and small data methods can help designers to study user needs more foundly and accurately. Taking kitchen storage products as an example, this paper firstly crawled the product reviews on the Tmall website using big data methods and the sentiment analysis of reviews was detected. Then with introducing an LDA model for topic mining, the reviews of users were transformed into the demand characteristic words of products. By small data methods, the implicit needs of users were obtained by questionnaires and in-home research, and the results of big data methods was Validated and supplemented. In this paper, the user needs indicators of four types of kitchen storage products were obtained and prioritized according to the kano model and sentiment analysis. The different needs of users in terms of living habits, staple food storage and kitchen utensils were illustrate according to the differences in Chinese food culture, thus providing design strategies for the future development of kitchen storage products.
\end{abstract}

Keywords. Large and traditional data, Emotional analysis, Kitchen-storage, implicit demand

\section{Introduction}

It is a new normal of Industry 4.0 that accelerates the industrial transformation relying on the information technology. With the rise of a variety of social networks as well as shopping platforms, the acquisition of user requirements is of particular significance, and various kinds of commodity comments turn into critical platforms to pass on and generate user requirements. Confronting complicated and diversified user requirements, there

${ }^{1}$ Corresponding Author, Qun Wu, 5 Second Avenue, Xiasha Higher Education Zone, Hangzhou, China; E-mail: wuq@zstu.edu.cn. 
have gradually been some weaknesses of the traditional small-data requirement analysis, including the time-lapse performance, low efficiency as well as strong subjectivity. The big-data analysis method on the basis of online evaluations is utilized in the mining process of user requirements. However, the online evaluations of products is often incomplete and needs to be crawled via digital tools, thus there exist certain technical barriers for the acquisition as well as analysis.

In accordance with the relevant literature on user requirements, it is found that scholars bring forward a vast majority of theories as well as methods, for example, through the clustering analysis, text mining, metaphor elicitation technique and other relevant methods, they analyze the online comments of products and explore user requirements. Luo puts forward explicit model as well as spiral model of explicit transfer of tacit knowledge on account of the user's tacit and explicit knowledge in product design, and predicts the research hot-spot issues as well as trends of the user's tacit knowledge in product design [1]. Chang develops the hierarchy of requirements via factor analysis, generates the implicit requirements of users by metaphor elicitation technique as well as the analysis of semantic differential, and finally creates a systematic mining method of user requirements [2]. Zhang probes into the evaluation data of the product performance dictionary, analyzes the connection between the positive as well as negative demand characteristics of evaluations and the types of user requirements, and laid down new theoretical foundation for the subsequent studies on user requirements [3]. Through the method of emotion analysis, Li obtains the explicit requirements of users, and the implicit requirements of users under the using scenario are acquired by scenario analysis [4]. On the basis of user-centric research concept, You carries on the survey of 14 separate-living users, deeply study the family environment of these people, and analyze the user requirements via in-depth survey [5]. By the videos and pictures, Kim M [6] keeps a record of the user's behavioral habits in the kitchen, and analyzes the user's behaviors as well as operational procedure after data acquisition.

The above studies furnish systematic guidance on the method for acquiring and analyzing user requirements, and make an attempt to probe into the user requirements from implicit and explicit perspectives. However, there is certain limitation of its research method, for it doesn't make a study on the user requirements from the angles of objective big data as well as subjective small data at the same time. Relying on the method of the acquisition as well as the analysis of user requirements via the integration of big and small data, this paper utilizes Python to crawl product evaluations for emotion analysis from the aspect of big data, and explores user requirement index of different emotional orientations via LDA model. In terms of small data, through the questionnaire as well as household survey, this paper acquires the diversified requirements of users in the context of different catering cultures, and analyzes the data from the questionnaires via SPSS software. The common and differential requirements of users are generated by the integration of big and small data, to fulfill the purpose of mutual verification and complementation between the two methods.

\section{A study of demand mining methods in the context of big and small data}

The concept of "big data" was first proposed by scholars at the end of the 20th century. Internet big data includes public data and media data, among which public data specifically refers to comments or other dynamic data uploaded by users on the Internet [7]. Big data analysis has been continuously advanced in product design, and its ability 
of trend analysis is gradually mature, which can help designers analyze users from a macro perspective. Small data is traditional data. Today, with the continuous development of science and technology, it is impossible to deeply obtain users' behaviors and attitudes only from the perspective of macro big data. Traditional data focuses more on the mining of personalized information, which can find users' hidden needs that big data cannot find. However, traditional data cannot be studied from a macro perspective if it is limited by the amount of data. It will be an inevitable trend to use the method of data fusion to study user needs. The comparison of its advantages and disadvantages is shown in Table 1.

Table 1. The advantages and disadvantages of big data and traditional data

\begin{tabular}{cll}
\hline Data type & \multicolumn{1}{c}{ Advantage } & \multicolumn{1}{c}{ Disadvantage } \\
\hline \multirow{3}{*}{ Big Data } & 1.High accuracy & 1.Difficulty in obtain information \\
& 2.High speed & 2.Unable to capture deep needs \\
& 3.Real-time information feedback & \\
\hline \multirow{2}{*}{ Small Data } & 1.Mining hidden demand & 1.Information delay \\
& 2.Simple in obtain information & 2.Small sample sizes \\
\hline
\end{tabular}

\subsection{Textual Sentiment Analysis}

Text sentiment analysis, also known as opinion mining and propensity analysis, uses text mining and computational linguistics to identify and extract subjective information from source materials. Text emotion can be divided into positive, intermediate and negative in the judgment of text emotion tendency. Under the background of the development of the digital economy, big data exist in the market demand, product development, product service at various stages, such as consumers through the network platform to complete the product purchase, and platform for shopping evaluation, evaluation is usually describe the multiple attribute dimension of product, can provide the market with a large number of valuable feedback information, clear user preferences for product features [8]. However, the lack of the user's emotional tendency can not enable the designer to judge the advantages and disadvantages of this feature. So it is necessary to determine the emotional orientation of the evaluation text before obtaining the feature words.

\subsection{LDA topic model modeling}

The concept of LDA model was first proposed by scholars Blei and David M in 2003, which used the co-occurrence relationship between words and words so as to infer the topic distribution of a document, and introduced the Dirichlet distribution of words and topics so as to discover the implied semantic dimensions from the text with an unguided learning approach, solving the fitting problem of traditional topic models, the principle of which is shown in Figure 1.

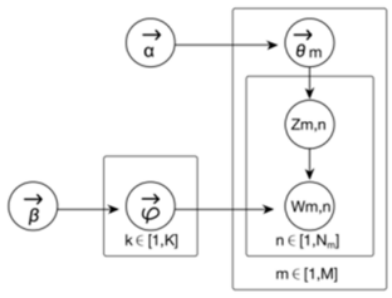

Figure 1.LDA model schematic diagram 


\subsection{Mapping principle of Kano model and emotion analysis}

Because different types of user needs have different effects on user satisfaction, user needs are divided into Attractive Quality, Performance Quality and Essential Quality based on the principle of KANO model Quality five elements Quality. According to the relationship between customer satisfaction and demand satisfaction degree, users' charm demand, expectation demand and basic demand can correspond to three kinds of user satisfaction: satisfaction, general and dissatisfaction. And it is mapped to positive feature words, neutral feature words and negative feature words in the sentiment analysis results. In Kano model, excitatory needs are generally distributed in favorable comments, expectant needs are generally distributed in neutral comments, and basic needs are mostly in bad comments.

\subsection{Research on small data methods}

Small data includes traditional methods such as questionnaire survey, household survey and user interview to obtain user needs. In order to have a deeper understanding of the target population, this paper first puts forward the corresponding research hypothesis through the research literature, then conducts the user positioning through the questionnaire survey, and then finds out the typical users in the household survey according to the results of the questionnaire survey and user interview, and excavates the discrepant needs of users.

\section{Demand model construction based on a fusion of data approach}

User demand mining can be divided into two stages: big data explicit demand mining and small data implicit demand mining. The integration of big data method and small data method can mine deeper implicit demand of users on the basis of obtaining common demand of users. In terms of big data, it is necessary to build a text mining model to get the frequency of keywords in the review text and the correlation degree of a key topic. In terms of small data, traditional research methods should be used to collect user characteristics, crowd information and specific behaviors and analyze the different needs of users. The specific steps are as follows:

The first step is to obtain the evaluation text. It is necessary to use crawler to crawl the evaluation data of target products from online shopping platform, and preprocess the data, and get the comment data set by deleting the characters of the comment variables. The second step is data cleansing. You first need to explore missing values in the data, clean up the data, then use the Snownlp module to split the text, and finally process common words in the string. The third step is to comment on the sentiment score . After calling the function to classify each comment, you need to connect to the Python sentiment rating database, calculate the probability average of each comment, determine the tendency of each comment text based on the probability average of the comment, and define the positive and negative tendency criteria. The fourth step is the topic selection of LDA model. After completing the classification of emotional tendency, each data set was divided into three categories: favorable, medium and bad reviews, and LDA model was constructed for each category in turn, subject selection and word frequency statistics were carried out. The fifth step is the visualization of data results. After the introduction of LDA model modeling, LDA model results of high, medium and low score data sets 
can be obtained, and the characteristic words of evaluation texts under different emotional tendencies can be obtained. The model results can be visualized by word cloud map, and then user demand indicators for this product category can be established. The sixth step is questionnaire survey, the specific steps can be divided into three stages: questionnaire design, questionnaire distribution and questionnaire result analysis. The data results of the questionnaire survey need to use SPSS statistical software for one-way analysis of variance, and determine the objects of household survey by mining the different needs of users. The seventh step is In-home research. This is a qualitative analysis of the selected users, which requires communication with the users before the research, and the use of tripods, cameras, etc. to record user behaviour during the household research in order to facilitate the decomposition of the recorded video at a later stage.

\section{Example study of kitchen storage product based on a data fusion approach}

Kitchen receive China starts relatively late, most choose to directly use abroad to receive way, but the Chinese food culture, regional characteristic, have much difference with foreign existing most receive product did not resolve our country kitchen receive product without systemic classification and regional kitchen utensils and appliances of different problems, are generally lower user satisfaction. Tmall platform is one of the largest online shopping platforms in China, and its product review area has gradually become a platform for consumer dialogue and communication. Therefore, this paper selects kitchen storage products as an example to obtain their user review data on Tmall platform, and obtains users' explicit demand and implicit demand based on the method of data fusion.

\subsection{Obtain evaluation text of Kitchen-storage Products}

Kitchen storage products can be roughly divided into three categories: food storage, operating equipment and kitchen appliances. In this paper, we mainly start with food storage and operating equipment products, choosing rice buckets, spice racks, dish racks and floor racks as representatives of kitchen storage products, and using python software to obtain reviews of the four products. The specific steps are as follows: (1) Confirm that Tmall shops- Ju Jiajia, a kitchen e-commerce platform with annual sales of over 300 million, is the source of data for these four products. (2) Search the four confirmed kitchen storage products on Tmall and select the product with the highest sales volume. (3) Using URL rules to intercept the user evaluation data of these four products, the textual content of the evaluation data mainly included colour, style and users' subjective thoughts.

\subsection{Kitchen-storage product evaluation data cleaning}

The buyer's nickname, short comments and other irrelevant data in the evaluation text were removed, and the final 4543 valid comments data were imported into Python software to explore missing values. Then, Snownlp module was used to segment the text and complete the processing of common words in strings. 


\subsection{Kitchen-storage product reviews emotional rating}

The probability mean of each comment of the four products was calculated by connecting python sentiment rating database, and the tendency of each comment text was judged according to its probability mean. Sentiment analysis can divide the evaluation data into three categories: good, medium and poor. By observing the data of comments in each range, it can be concluded that most of the comments with a score lower than 0.3 are poor. The rectangle scoring chart of rice barrel shows that there is a big difference in emotional score at 0.4 , and the negative rating is high. The visual score of the condiment rack data set showed that there was a big difference in the comments at 0.6. Combining the emotional score distribution of the ground shelving and dish rack, it was confirmed that the score below 0.3 was negative, the score between 0.3 and 0.7 was neutral, and the score above 0.7 was positive. The bar chart of product sentiment analysis is shown in Figure 2 (taking floor shelving and dish rack as examples).
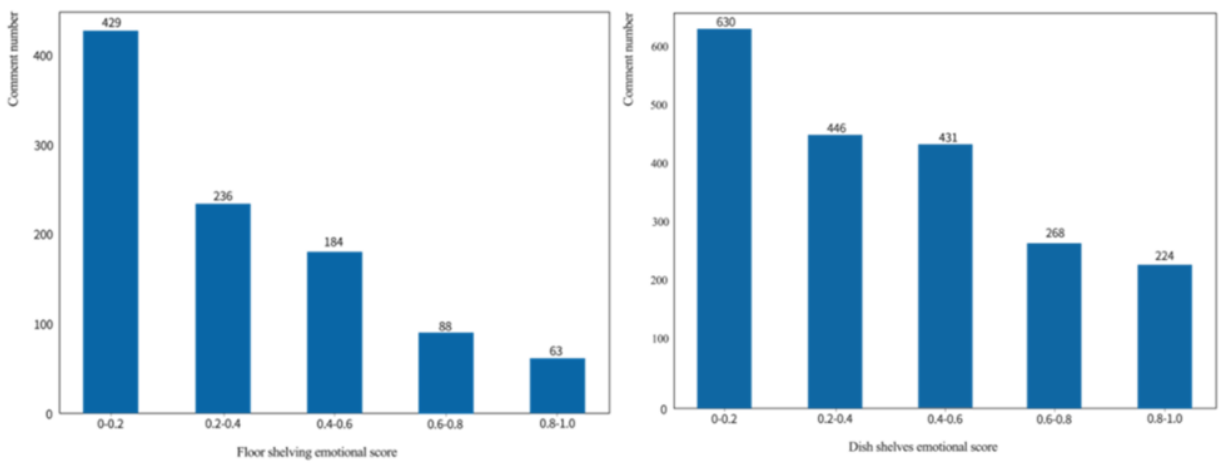

Figure 2. Product sentiment score bar chart

\subsection{Introduction of LDA model for kitchen-storage products}

After classifying the evaluation texts of four categories of kitchen products, LDA model was introduced to select the theme and count the word frequency, and the feature words and their weights under different emotional tendencies of each category of products were obtained. Considering that the factors affecting user satisfaction are mainly concentrated in the negative review text, the low-rating data sets of four kitchen storage products are mainly analyzed.

From the results of the LDA model, it can be seen that when the number of topics is set to 1 , the three highest weighted negative feature terms in the rice bucket low rating dataset are odour, sealability and material durability, where the weight value of odour is 0.033 , sealability is 0.028 and material is 0.024 . It can be seen that the most influential factor in the rice bucket low rating dataset is the product odour. In the low score data set of ground shelving, the influencing factors of products mined by LDA model are logistics, wheels and space, among which the weight value of logistics is 0.041 , which is the biggest influencing factor of negative comments of ground shelving. In the low-score data set of condiment rack, the influential factors of high weight words are deformation, unbalance and logistics. Finally, in the low-score data set of dish rack, the characteristic words are accessories, space and logistics respectively, among which the weight value of screws as accessories is 0.079 , accounting for the highest weight. 


\subsection{Analysis of macro data results}

The results of the above LDA model were visualised in the form of word cloud diagrams, as shown in Figure 3 (taking the rice bucket product feature words as an example). The negative, neutral and positive sentiment words were classified, and based on the mapping principle of Kano model and sentiment analysis mentioned above, the words were transformed into the specific needs of users, and the basic, desired and charming needs indicators of users were constructed respectively to form a user needs indicator chart, as shown in Figure 4.

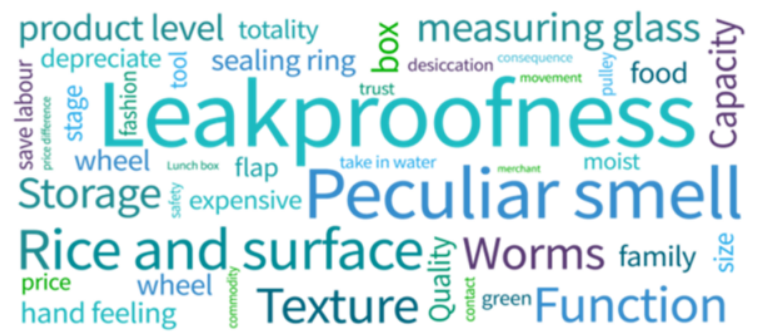

Figure 3. Rice bucket word cloud image

\begin{tabular}{|c|c|c|c|c|c|c|}
\hline Product Vame & Negathe feature mord & Bask demand indicators & Neutral leature word & Performance demand indicators & Negative feature word & Atrractive demand indicate \\
\hline \multirow{3}{*}{ Floor shelving } & Wheel & Redesign of the wheel structure & Partition & Self-adjustable Partition & Texture & Focus on product surface textu \\
\hline & Logistics & Shockproof logistics packaging & Delivery & Speed the delivery up & Function & Functional diversification \\
\hline & Space & Targeted space design & Plastic & More durable plastic & Appearance & Beautify the appearance \\
\hline \multirow{3}{*}{ Spice rack } & Roughness & Material smoothness & Sire & Available in a varicty of sizes & Delicacy & Easy to store and move \\
\hline & Transshape & Stronger material & Logistics & Improve logistics efficiency & Space & Reasonable space setting \\
\hline & Waterdamage & Avoid bacteria from standing water & Senice & High quality after-sales service & Appearance & Optimize appearance style \\
\hline \multirow{3}{*}{ Ricer box } & Peculiar smell & Safe and odor-free material & Dampproof & The inside of the product is kept dry & Texture & Adopt safe and durable materii \\
\hline & Gap & Tightness & Worms & Confined space & Capacity & Expand capacity \\
\hline & Rice and noodles & $\begin{array}{l}\text { Design for the storage of rice and } \\
\text { surface }\end{array}$ & Quality & Enhance quality & Appearance & Optimize product appearance \\
\hline \multirow{3}{*}{ Dish shelves } & Screw & Complete Kits & Function & Add additional features & Waterlogging & Quick dry \\
\hline & Undersize & Enlarge the sire & Dust & $\begin{array}{l}\text { Avoid dust accumulation in dead } \\
\text { spots }\end{array}$ & Style & Various styles \\
\hline & Plastic & Avoid plastic odor & Diameter & Increase product diameter & Detail & Enhanced control of detail \\
\hline
\end{tabular}

Figure 4. User demand indicator chart

\subsection{Questionnaire survey and result analysis}

The questionnaire survey mainly focuses on the current situation of kitchen storage, kitchen environment and user behavior. The purpose is to understand the satisfaction and behavioral differences of users on kitchen storage products under different food cultures, so as to obtain the relevant influencing factors of kitchen storage products behind the differences in food cultures between the North and the South. The author selected Shandong cuisine and Anhui cuisine as two of the eight major cuisines for the study.The questionnaire design was evaluated by three experts as valid and then distributed online. The basic user information is shown in Table 2 . 
Table 2. Basic User Information of questionnaire

\begin{tabular}{|c|c|c|c|c|c|c|c|}
\hline Index & category & frequen & proportion & Index & category & frequency & proportion \\
\hline \multirow{2}{*}{ area } & $\begin{array}{c}\text { Anhui } \\
\text { Province }\end{array}$ & 118 & $52.70 \%$ & \multirow{5}{*}{$\begin{array}{l}\text { population of } \\
\text { family }\end{array}$} & 2 & 18 & $8.00 \%$ \\
\hline & $\begin{array}{l}\text { Shandong } \\
\text { Province }\end{array}$ & 106 & $47.30 \%$ & & 3 & 93 & $41.50 \%$ \\
\hline \multirow{2}{*}{ gender } & male & 90 & $40.20 \%$ & & 4 & 61 & $27.20 \%$ \\
\hline & female & 134 & $59.80 \%$ & & 5 & 38 & $17.00 \%$ \\
\hline \multirow{4}{*}{ age } & $<35$ & 79 & $35.30 \%$ & & $>5$ & 14 & $6.30 \%$ \\
\hline & $46-55$ & 81 & $36.20 \%$ & \multirow{3}{*}{$\begin{array}{c}\text { Share kitchen } \\
\text { chores }\end{array}$} & cooperation & 201 & $89.70 \%$ \\
\hline & $56-60$ & 9 & $4.00 \%$ & & undertakes alone & 23 & $10.30 \%$ \\
\hline & $>60$ & 3 & $1.30 \%$ & & & & \\
\hline
\end{tabular}

By means of SPSS software, this paper carries through one-way analysis of variance on the questionnaire results, and explores the differences between the users in Anhui and Shandong. The followings are the concrete contents: As for the humidity of kitchen environment in Shandong and Anhui, there are $42 \%$ and $19 \%$ respectively for the perennial aridity ratio, $36 \%$ and $57 \%$ respectively for the moist ratio of rainy season, as well as $22 \%$ and $24 \%$ respectively for the perennial moist ratio. By one-way analysis of variance, it is found that the Sig value (significance) of kitchen humidity between the two regions is $0.02<0.05$, which is of significant differences. The overall humidity of kitchen environment in Shandong is lower than that in Anhui, while the kitchen environment in southern China is more humid with the example of Anhui. Therefore, close attention shall be paid to the moisture-proof and mildew-proof performance for the kitchen storage. In regard to the selection of staple food, $65 \%$ of users take wheaten food as their staple food in Shandong while only $8 \%$ in Anhui. The Sig value of the selection of staple food between the two regions is $0.000<0.05$. Rice is the staple food in Anhui, while wheaten food is more popular in Shandong. The Sig value of the storage of preserved meat between the two regions is $0.001<0.05$. People usually make dried goods and store them at home in Shandong. Due to the dry climate in northern China, the dried food can be well-preserved. Due to the rainy and humid climate of the cities in southern China, although there are some families to make preserved meat themselves, it cannot be well-preserved.

\subsection{Household survey and result analysis}

Household survey refers to qualitative analysis of typical users in two regions, and mining users hidden needs through on-the-spot observation of users behaviors. six households are selected from the questionnaire survey object for household investigation, including three households in Shandong and Anhui respectively. The specific information of users is shown in Table 3 and Table 4. 
Table 3. Shandong User Information of household survey

\begin{tabular}{|c|c|c|c|c|}
\hline $\mathrm{Nan}$ & Rogion und Ponike & $\mathrm{Ag}$ & Femaly informution & 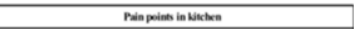 \\
\hline \multirow{5}{*}{ Mns Bung } & \multirow{5}{*}{ Humphem Antui provine } & \multirow{5}{*}{$s$} & Four people & \multirow{5}{*}{ 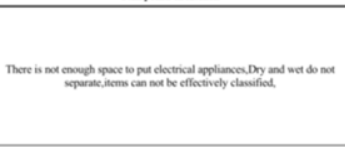 } \\
\hline & & & 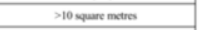 & \\
\hline & & & 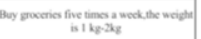 & \\
\hline & & & Hewir tate & \\
\hline & & & Risce & \\
\hline \multirow{5}{*}{$M+L$} & \multirow{5}{*}{ Humpenem Mnthi porime } & \multirow{5}{*}{$\$ 0$} & Threx popic & \multirow{5}{*}{ 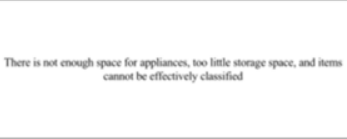 } \\
\hline & & & 7.10 squere maters & \\
\hline & & & 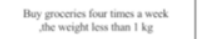 & \\
\hline & & & spiy food lorers & \\
\hline & & & Rise & \\
\hline \multirow{4}{*}{ Mas Weng } & \multirow{4}{*}{ 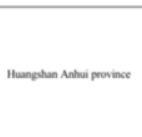 } & \multirow{4}{*}{$s$} & 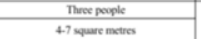 & \multirow{4}{*}{ 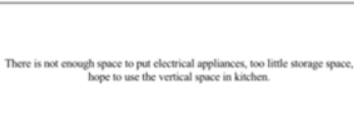 } \\
\hline & & & 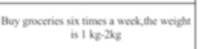 & \\
\hline & & & Bland dist & \\
\hline & & & Rise & \\
\hline
\end{tabular}

Table 4. Anhui User Information of household survey

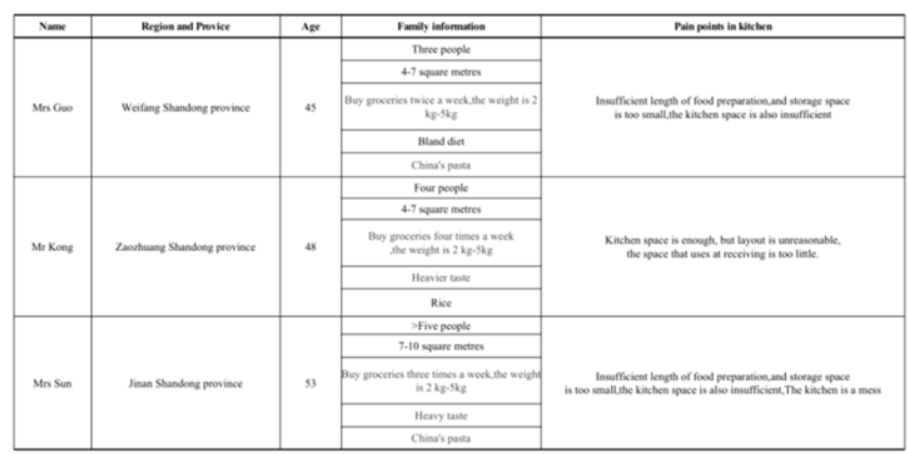

After the user sample database was established based on the results of household investigation, Ms. Guo from Shandong Province and Ms. Zhang from Anhui Province were respectively taken as examples to analyze the household dishes, items stored in the kitchen and behavioral activities, and conclude the pain points in the cooking process of users. Shandong kitchen features and pain points which were as follows: in addition to the characteristics of kitchen utensils and storage issues, in terms of diet, Ms. Guo a light diet, staple food is given priority to with pasta, making dumplings and steamed buns at large operating space, because the kitchen mesa area is lesser, users often can only go to other area to make pasta. In the process of investigating Ms. Zhang in Anhui Province, Ms. Zhang's family likes to eat spicy food and a small amount of characteristic homemade pickled food. Kitchen area of Miss Zhang home is bigger, added to buy content ark to be used at placing the article such as pot to provide on original space foundation, but the metope space that did not use the kitchen undertakes receiving. Due to the differences of climate, Anhui area rainfall all year round, and environmental humidity, kitchen utensils and appliances need to put in the balcony after drying and storage of food (rice and dry goods) also easy be affected with damp be affected with damp mildew to change, so in Anhui area kitchen receive product especially need to consider the degree of sealing and food storage. 


\section{Conclusion and outlook}

Relying on the method of the acquisition of user requirements via the integration of large and small data, this paper develops a data-mining framework for user requirements by means of the integration of large and small data, and with the case study on the kitchenstorage products, it successfully probes into the common requirements of users for the kitchen-storage products as well as the diversified requirements of users in the context of different catering cultures. On the one hand, small-data method plays a role of the complementation to the common requirements of users acquired by big-data method, and explores the implicit requirements, including the differences of catering culture, the population differences as well as the storage of local specialty food. On the other hand, large-data method also provides scientific evidence for the effectiveness of small-data method. For example, through small-data research, it was found that due to the moist climate in Anhui Province, the kitchen utensils as well as the staple food stored is easy to mildew and needs to have sun exposure frequently, thus there is a high requirement for the sealing performance of the products; it is also verified by big-data mining, that is, the demand characteristics of the rice bucket mined by LDA model are the sealability and moisture-proof performance.

To this end, there is mutual verification as well as mutual complementation between the big-data and small-data method, and only on the basis of big data and traditional data simultaneously can we integrate their strengths and avoid their respective weaknesses. The integration of big and small data enables the acquisition of user requirements to no longer remain on the superficial level, but explores in-depth implicit requirements of users while acquiring the explicit requirements of users, so as to provide a reference for the development direction of future product design, and essentially perfect the user experience.

\section{Acknowledgment}

This work is supported by Zhejiang Provincial Key Laboratory of Integration of Smart Kitchen System(Grant No: 19080049-N)

\section{References}

[1] Luo, S. J., et al. Statues and progress of research on users' tacit knowledge in product design. Computer Integrated Manufacturing Systems 16.4(2010):673-688.

[2] Li, X. L. , Chang, S. X. User Requirements Mining and Case Study in Custom Wardrobe Design. Packaging Engineering 42.12(2021):135-141

[3] Zhang, W. X. , et al. Review Data Driven Customer Need Model Research Based on Product Performance.Chinese Journal of Mechanical Engineering 31.15(2020):1866-1876.

[4] Li, X. , et al. Research on Product User Demand Insight Method Based on SPSS and Online Comment Analysis. Packaging Engineering .

[5] You, et al. Design Concept Study of Kitchen Utensils for Single Household. Journal of Digital Design 15.3(2015):19-26.

[6] Kim, et al. A Study on Kitchen Use Behaviors through User Observation - Focused on Small-sized Apartments in Shanghai, China -." Journal of the Korean Institute of Interior Design (2015).

[7] Rathore, M. Mazhar, et al. Urban planning and building smart cities based on the Internet of Things using Big Data analytics. Computer Networks the International Journal of Computer \& Telecommunications Networking 101.C(2016):63-80.

[8] Zhao, Z., et al. Research on Dimension Mining and Sentiment Analysis for Chinese Product Comments. 